\title{
PATRONES DE PERSONALIDAD, SÍNDROMES CLÍNICOS Y BIENESTAR PSICOLÓGICO EN ADOLESCENTES
}

\author{
MARÍA M. CASULLO ${ }^{1}$ y ALEJANDRO CASTRO SOLANO ${ }^{2}$ \\ ${ }^{1}$ Universidad de Buenos Aires, Argentina \\ ${ }^{2}$ Universidad de Palermo, Buenos Aires, Argentina
}

(Aceptado en noviembre de 2001)

\begin{abstract}
El presente trabajo analiza dimensiones del bienestar psicológico y patrones de personalidad en estudiantes adolescentes con y sin presencia de síntomas y síndromes clínicos. Las pruebas SCL-90-R y MACI, y una escala de bienestar psicológico, fueron administradas a una muestra de 305 estudiantes adolescentes de escuelas públicas argentinas. Se verifić que el $27 \%$ presentaba síntomas y síndromes de relevancia clínica, especialmente referidos a depresión, tendencias suicidas y trastornos alimentarios. Los adolescentes de riesgo alto (RA) percibían menor bienestar psicológico, especialmente en las dimensiones proyectos de vida y control de situaciones. Por su parte, aquéllos de riesgo bajo (RB) tendían a dramatizar sus conflictos, eran más individualistas y conformistas, revelaban mayor tendencia a la insensibilidad social y parecían estar más disconformes con su sexualidad.

Palabras clave: Bienestar psicológico, adolescentes, patrones de personalidad, síndromes clínicos.
\end{abstract}

Personality patterns, clinical syndromes and psychological well-being in adolescents

Dimensions of psychological well-being in adolescents with and without presence of clinical symptoms and personality patterns were analysed. For assessment purposes, SCL-90-R, MACI and a measure of psychological well-being (BIEPS) were administered to a sample of $\mathbf{3 0 5}$ adolescent students attending public schools in Buenos Aires city. We found presence of different kinds of psychopathology in $27 \%$ of the total sample. Psychological well-being was lower in subjects with clinical patterns and symptoms, mainly in the domains related with life projects and control of events. Subjects considered not being at risk showed tendencies to dramatize, were unruly and conforming and expressed concerns related to sexual discomfort and social insensitivity.

Key words: Psychological well-being, adolescents, personality patterns, clinical syndromes.

\section{INTRODUCCIÓN}

Tradicionalmente la adolescencia ha sido conceptuada como un período de carencias y necesidades, una época de tormentas y malestares en la que aparecían determinadas alteraciones conductuales, cognitivas y afectivas no consideradas patológicas (Aberastury y Knobel, 1971). En las últimas dos décadas del siglo $\mathrm{xX}$ los investigadores han enfocado su estu-

Correspondencia: María M. Casullo, Facultad de Psicología, Universidad de Buenos Aires, Independencia 3065, 1225 Buenos Aires, Argentina. Correoe: cesullo@psi.uba.ar dio como una etapa de transición en el ciclo vital personal en la que se producen hechos de alta relevancia para el logro de una vida adulta sana: los cambios corporales relacionados con la pubertad, el acceso a la sexualidad reproductiva, el establecimiento de nuevos vínculos psicosociales, en especial con personas de sexo diferente al propio, el logro de ciertas competencias intelectuales que hacen posible una manera diferente de pensar y analizar la realidad, la construcción de las identidades sexuales y ocupacionales. En la cultura occidental urbana se ha verificado una prolongación en años de 
la duración de esta etapa, por lo que autores como Elliott y Feldman (2000) sugieren hablar de sub-etapas: la adolescencia temprana (11 a 14 años), la media (15 a 17 años) y la adolescencia tardía (18 a 25 años).

Hill (1993) ha revisado una serie de estudios empíricos sobre adolescentes y considera que es un período evolutivo en el que sólo algunos individuos desarrollan problemas con significación clínica. Las transiciones que tienen lugar durante la segunda y tercera décadas de la vida de todo individuo proveen las estructuras y recursos que permiten que el niño se transforme en adolescente y éste en un adulto (McCullough et al., 2000). Todas las transiciones tienen en común un elemento de discontinuidad, que si bien no necesariamente es abrupta o azarosa, implica un proceso de cambio en la manera en la que las personas se autoperciben y perciben el mundo en el que están inmersas; todo cambio siempre implica un riesgo. Las experiencias de transición (la pubertad, el ingreso a la escolaridad media, la finalización de esos estudios, construir la identidad sexual) alteran el equilibrio afectivo y la percepción subjetiva de bienestar. Como bien lo señalan Petersen y Leffert (1995) la adolescencia se caracteriza por experiencias de cambios, es un período de desafíos no necesariamente negativo y problemático, a menos que condiciones socioculturales o neurobiológicas específicas lo provoquen. Las principales transiciones que se llevan a cabo durante la adolescencia suponen un movimiento hacia comportamientos más libres y responsables; junto a los cambios puberales y cognitivos se producen modificaciones en el ámbito de las afiliaciones (los vínculos con los padres, los pares, las experiencias de enamoramiento), en el campo de la actividad académica-laboral (la escuela y el mundo del trabajo) y se construye la identidad personal. El recorrido de esa transición está condicionado por las posibilidades biológicas, personales y culturales y afecta el logro de la salud psíquica, entendida como la percepción subjetiva y objetiva de bienestar (Lemos y col., 1992). Este logro es posible cuando las metas que el sujeto se propone alcanzar armonizan con sus desarrollos físico, afectivo, cognitivo y social y sus condiciones de vida (Schulenberg et al., 1997). Una persona tiene un alto bienestar psicológico (BP) si experimenta satisfacción con su vida, si su estado anímico es bueno (afectos positivos) y sólo ocasionalmente experimenta emociones poco placenteras como tristeza, rabia, inhibiciones (afectos negativos) (Fierro y Cardenal, 1996). La percepción de BP puede ser considerada como el componente cognitivo de la satisfacción vital ya que implica valoraciones que las personas hacen respecto a cómo les ha ido o cómo les está yendo en el transcurso de sus vidas (Diener y Suh, 2001). El análisis de los factores asociados con el BP es una buena manera de comprender su significado, teniendo presente que esos factores varían en función de las diferencias culturales. Tal como afirman los autores antes mencionados, las emociones son buenos predictores del $\mathrm{BP}$ en las denominadas culturas individualistas mientras que en las colectivistas el BP está mucho más asociado con las conductas de respeto y cumplimiento de las normas sociales vigentes. Por eso es necesario destacar que el BP puede ser analizado en dos niveles: sociocultural (producto bruto interno per capita, promedio de expectativa de vida, tasas de desempleo) e individual (actitudes, opiniones, creencias, percepciones). En el presente trabajo se aborda su análisis desde la percepción subjetiva que tienen los adolescentes, sobre la base de las propuestas de Ryff y Keyes (1995) y Ryff (1989) quienes sostienen que el constructo se operacionaliza en seis dimensiones: aceptación de sí mismo, relacio- 
nes positivas con los otros, autonomía, control del medio ambiente, construcción del proyecto de vida y crecimiento personal.

Interesa poder discriminar entre los comportamientos, emociones y vivencias subjetivas que son expresión de crecimiento y cambio y los que implican disfunción y/o patología. Tradicionalmente se ha definido a los adolescentes en riesgo en función de determinadas variables sociodemográficas, personales o familiares, como la pertenencia a un grupo de bajo nivel sociocultural o el bajo nivel educativo (Mc Whiter, Mc Whiter y Mc Whiter, 1993; Pallas, Natriello y McDull, 1989). En términos generales cabe afirmar que los adolescentes tienen cantidad suficiente de información acerca del riesgo implícito en muchas de sus acciones a pesar de lo cual éstas no la reflejan. Estar informado no significa que siempre se actúe teniendo en cuenta esa información (Frost, 1992). Beck (1992) sostiene que varias de las características asociadas con los comportamientos adultos sanos (trabajo estable, vida familiar) se han fragmentado de tal manera en la sociedad urbana actual que generan en los adolescentes una flexibilidad emancipadora y sentimientos de ansiedad $\mathrm{e}$ incertidumbre; el comportamiento de riesgo surge como una solución transitoria aparente. Walker y Towsend (1998) informan sobre la existencia de altos porcentajes de adolescentes con problemas emocionales de gravedad: el $15 \%$ en población general no consultante. Aproximadamente el $50 \%$ de ese total está conformado por casos nuevos; el $50 \%$ restante es una prolongación de problemas ya presentes en la infancia. Muchas alteraciones emocionales de la infancia cristalizan en la adolescencia convirtiéndose en características disfuncionales estables de personalidad (Bernstein et al., 1996). ¿Por qué algunos jóvenes desarrollan trastornos psicológicos y otros que atraviesan las mismas cir- cunstancias vitales resultan indemnes a tal situación? Muchos adolescentes no desarrollan patologías a pesar de estar expuestos a situaciones vitales estresantes.

Ciertos autores diferencian los conceptos de vulnerabilidad y riesgo. Para Ingram y Price (2001) la vulnerabilidad está asociada con la causalidad, alude a los mecanismos relativamente estables (hereditarios o sociales) productores de un cuadro o trastorno patológico. Estos mecanismos pueden estar presentes en los subsistemas cognitivo, afectivo, fisiológico, social o cultural que conforman al sujeto humano en su condición de sistema complejo e interactúan de forma dinámica y recíproca. La psicopatología es la resultante de fracasos o dificultades en el proceso de negociación que supone el empleo adecuado de recursos de los subsistemas mencionados en la resolución de problemas específicos (Cicchetti y Cohen, 1995) en el transcurso del ciclo evolutivo. Los factores de riesgo, por su parte, se refieren a variables correlacionales y descriptivas empíricamente asociadas con la manifestación de determinadas patologías. Conocer el factor de riesgo permite predecir la probabilidad de que una disfunción o trastorno pueda estructurarse o manifestarse. Es importante no confundir causa con ocurrencia. Algunos investigadores dan importancia al estudio de variables relacionadas con lo opuesto a la vulnerabilidad: la invulnerabilidad, asociada con la competencia, los factores protectores, el sentido de coherencia, los mecanismos de resistencia (resilience) que impiden la estructuración de la patología, sin suponer inmunidad.

Es necesario entonces estudiar el impacto de los factores de resistencia que neutralizan el efecto de los estresores: fortalezas constitucionales, historias familiares sanas, el apoyo social, los hábitos de salud, las disposiciones de personalidad (Cobaza et al., 1979). Durante 
mucho tiempo la psicología se ha ocupado más de estudiar los aspectos patológicos, enfermos y desajustados relacionados con el malestar (Veenhoven, 1988) descuidando los aspectos generadores de salud. Desde fines de la década del 80 se han incrementado las propuestas psicológicas que debaten un nuevo paradigma al que denominan psicología positiva o "salutogénica» (Casullo, 2001). Los individuos más felices y satisfechos sufren menos malestar, tienen mejores autoconceptos, un mejor dominio del entorno y poseen buenas habilidades sociales. La satisfacción y el bienestar psicológicos podrían caracterizarse como indicadores de un buen funcionamiento mental (Argyle, 1987). Veenhoven (1995) afirma que los individuos felices superan en una proporción de 3 a 1 a los que no lo son y caracteriza la felicidad y el BP como disposiciones bio-psicológicas naturales. El bienestar subjetivo es el equivalente psicológico del concepto biológico orgánico de salud.

¿Qué factores posibilitan que ciertos adolescentes no desarrollen trastornos psicológicos aunque todo indicase que ello es probable? Las investigaciones sobre adolescentes en riesgo que venimos desarrollando desde el año 1990 en escuelas públicas de la ciudad de Buenos Aires han permitido aislar algunos de esos factores protectores: posibilidad de pensar en un proyecto de vida, criterio de realidad o sentido común, tolerancia a la frustración, presencia de redes de apoyo afectivo, baja impulsividad, percepción de bienestar subjetivo (Casullo et al., 2000).

El objetivo general del siguiente estudio es analizar los factores asociados a la dimensión subjetiva bienestar psicológico en adolescentes. Esto nos permitirá discriminar de forma objetiva entre adolescentes en riesgo alto de sufrir algún tipo de psicopatología o en riesgo bajo y, de esta forma, actuar en el contexto de las instituciones educativas para la identificación temprana de trastornos en adolescentes.

De forma más concreta, pretendemos señalar los siguientes objetivos específicos que se desprenden del objetivo general: Analizar y comparar percepciones subjetivas de BP en estudiantes que presentan síntomas psicopatológicos y síndromes clínicos, estimados como de riesgo alto (RA) y en aquéllos considerados de riesgo bajo (RB). Específicamente, mediante el presente estudios se pretende: (a) Analizar las relaciones probables entre la percepción subjetiva de BP y los patrones de personalidad según las propuestas de T. Millon; (b) Determinar si existen diferencias significativas según sexo y edad en relación con los diferentes agrupamientos (clusters) psicopatologicos; (c) Obtener datos empíricos que permitan proponer la evaluación de la percepción subjetiva del BP como estrategia para la identificación temprana de adolescentes en riesgo en el contexto de instituciones educativas.

\section{MÉTODO}

\section{Participantes}

Participaron en el estudio 305 adolescentes, varones y mujeres, de 13 a 17 años de edad, alumnos de institutos de educación secundaria ubicados en la ciudad de Buenos Aires. Para seleccionar a los adolescentes de riesgo alto (RA) se tomaron en cuenta las puntuaciones del SCL-90-R. Siguiendo el criterio propuesto por Derogatis (1994) se consideró caso clínico al que puntuaba por encima del percentil 84 en el índice general de sintomatología (GSI) y/o obtuvo una puntuación superior al percentil 84 en por lo menos dos de las escalas primarias del listado de síntomas. Mediante este procedimiento se ubicó en la categoría "caso clínico» (RA) a 83 estu- 
diantes de los 305 que participaron en el estudio. Se tomó como grupo control $(n=$ 222) a los estudiantes que habían puntuado bajo en el SCL-90-R, no considerados casos clínicos (i.e., de RB). La media de edad, para el total de casos analizados ( $55 \%$ mujeres y $45 \%$ varones) era de 14,71 para las mujeres $(D T=1,49)$ y de 15,04 para los varones $(D T=1,34)$. Los participantes pertenecen a un nivel socioeconómico medio y medio-bajo, calculados considerando el nivel ocupacional y educacional de los padres o adultos a cargo de los adolescentes.

\section{Pruebas psicológicas}

Cuestionario SCL-90-R (Symptom Checklist-90-Revised; Derogatis, 1994). Se utilizó la versión argentina de (Casullo y Castro Solano, 1999). Es un cuestionario de autoinforme de 90 síntomas. Los síntomas están agrupados en 9 áreas: ansiedad, depresión, ideación paranoide, psicoticismo, somatización, obsesiones y compulsiones, sensibilidad interpersonal, ansiedad fóbica y hostilidad. El sujeto tiene que responder si le molestó o preocupó cada síntoma durante la ultima semana, en una escala tipo Likert con 5 opciones de respuesta (nada a mucho). El cuestionario permite la obtención de tres índices globales que informan, en una puntuación única, sobre el nivel de profundidad y dimensión de malestar psicológico: (1) Índice de severidad global (GSI); es un indicador del nivel actual de gravedad del desorden; combina el número de síntomas y la intensidad del estrés percibido. (2) Indice de malestar sintomatológico; mide la intensidad de los síntomas e informa si quien responde los exagera o atenúa. (3) f́ndice de síntomas positivos; indica la cantidad de síntomas informados; es una medida de la cobertura de los síntomas en relación con diferentes áreas.
Cuestionario MACI (Millon Adolescent Clinical Inventory; Millon, 1993). Es un cuestionario de autoinforme que evalúa patrones de personalidad, áreas de expresión de preocupaciones e identifica aquellos síndromes clínicos más prevalentes en los adolescentes. Está integrado por 180 items que se responden por verdadero o falso. Se utilizó para este estudio la versión argentina de Casullo et al. (1998, 1999). Está compuesto por 3 secciones: (1) Patrones de personalidad; doce escalas evalúan características de la personalidad (introvertido, inhibido, afligido, sumiso, dramatizador, egoísta, transgresor, sádico, conformista, oposicionista, autodegradante y tendencia borderline). (2) Preocupaciones expresadas; ocho escalas se centran en sentimientos y actitudes sobre temas que conciernen a la mayoría de lo adolescentes: difusión de identidad, autodesvalorización, desaprobación corporal, disconformidad sexual, inseguridad grupal, insensibilidad social, discordia familiar y abuso infantil. (3) Síndromes clínicos; las siete escalas que componen esta sección se refieren a desórdenes que se manifiestan de manera específica: afecto depresivo, abuso de sustancias, disfunciones de la alimentación, tendencia al uso de sustancias, predisposición a la delincuencia, tendencia a la impulsividad, sentimientos de ansiedad y tendencia suicida. El inventario presenta cuatro índices reveladores que indican el estilo de respuesta del paciente (sinceridad, deseabilidad, depreciación y fiabilidad).

Escala de bienestar psicológico (BIEPS; Casullo, 1999). Es una escala de autoinforme de 13 items que evalúa el bienestar psicológico (BP) sobre la base de las cuatro dimensiones siguientes: (1) control de situaciones, (2) vínculos psicosociales, (3) proyectos de vida y (4) aceptación de sí mismo. Está basada en la teoría de la satisfacción elaborada por Ryff y Keyes (1995), Ryff (1989), y Schmutte y Ryff (1997). 


\section{Procedimiento}

El cuestionario SCL-90-R y la escala BIEPS fueron administrados grupalmente en las instituciones educativas, en el horario en el que se imparten las clases. En función de las puntuaciones obtenidas en el SCL-90-R fueron seleccionados los estudiantes considerados "casos clínicos" o de riesgo alto (RA). A estos adolescentes se les administró el MACI en forma grupal (grupos integrados por 5 a 7 personas). También se administró el MACI a estudiantes con puntuaciones bajas [riesgo bajo (RB) o grupo control]. Se informó a los participantes que sus respuestas serían tratadas de modo confidencial. La escuela no tuvo conocimiento de los resultados individuales de cada participante. Los adolescentes detectados como "casos clínicos» fueron citados para una entrevista clínica individual, tarea que permitió confirmar la categorización diagnóstica basada en las puntuaciones de la prueba MACI en el 93\% de los casos. A partir de entrevistar a uno o ambos padres fueron concretadas derivaciones a un centro de salud comunitario. Es importante destacar que ninguno de los adolescentes entrevistados había recibido o estaba recibiendo asistencia psicológica o psiquiátrica al momento de ser evaluados. Los datos presentados forman parte de un estudio más amplio sobre factores de riesgo y psicomorfología de la salud al que ya se ha hecho referencia.

\section{RESULTADOS}

\section{Comparación de perfiles de personalidad}

Se compararon las puntuaciones en el MACI de ambos grupos de jóvenes (RA y RB). Los resultados muestran que los adolescentes RA obtuvieron altas puntuaciones en la mayoría de las escalas del
MACI (Tabla 1). No se encontraron diferencias significativas en 4 de esas escalas: sumisión, insensibilidad social, predisposición a la delincuencia y ansiedad. Los adolescentes considerados en RA muestran un patrón de personalidad más desajustado y con características borderline en comparación con aquéllos considerados RB. Presentan alteraciones psicopatológicas tanto de tipo internalizantes (introvertido, inhibido, triste y autodestructivo) como externalizantes (indisciplinado, sádico, oposicionista). Las preocupaciones más frecuentes están relacionadas con la autoimagen y la autopercepción (difusión de identidad, autodesvalorización, desaprobación corporal), la calidad de los vínculos con los pares y la familia (inseguridad social y discordia familiar) y el abuso infantil. Presentan también puntuaciones promedio más elevadas en los síndromes clínicos que aquéllos considerados RB. Los adolescentes de RB dramatizan más sus malestares, son más individualistas y conformistas, parecen más disconformes con su sexualidad y tienden a mostrar más insensibilidad social.

\section{Patrones de personalidad de estudiantes $R A$}

Para verificar los patrones diferenciales de personalidad disfuncional que tienen los estudiantes de RA se llevó a cabo un análisis de clusters de perfiles. Previamente se tipificaron las puntuaciones del MACI. Se tomó como criterio para interpretar una elevación en cada escala una desviación típica mayor a 1 . La mejor solución encontrada fue la de 4 clusters. Los grupos fueron caracterizados de la siguiente forma:

1) Grupo con trastornos internalizantes leves $(n=24)$. Estos adolescentes presentan déficits en la polaridad placer 
Tabla 1. Puntuaciones en el MACI de adolescentes con alto y bajo riesgo psicopatológico

\begin{tabular}{|c|c|c|c|c|c|}
\hline \multirow[b]{2}{*}{ Escalas MACI } & \multicolumn{2}{|c|}{$\begin{array}{c}\text { Bajo riesgo } \\
(n=222)\end{array}$} & \multicolumn{2}{|c|}{$\begin{array}{l}\text { Alto Riesgo } \\
(n=83)\end{array}$} & \multirow[b]{2}{*}{$t$} \\
\hline & Media & DT & Media & $D T$ & \\
\hline Apertura & 292.39 & 59.96 & 380.24 & 67.71 & $11.22^{\star}$ \\
\hline Deseabilidad & 10.64 & 2.76 & 8.56 & 3.28 & $5.72^{\star}$ \\
\hline Defensividad & 2.75 & 2.39 & 7.09 & 3.54 & $12.54^{\star}$ \\
\hline Introvertido & 20.18 & 7.37 & 29.71 & 9.75 & $9.39^{\star}$ \\
\hline Inhibido & 17.34 & 8.59 & 27.44 & 11.97 & $8.35^{\star}$ \\
\hline Triste & 9.57 & 7.76 & 21.80 & 9.59 & $11.74^{*}$ \\
\hline Sumiso & 47.73 & 9.12 & 47.44 & 13.18 & 0.21 \\
\hline Dramático & 40.55 & 7.73 & 33.36 & 10.55 & $6.67^{\star}$ \\
\hline Egoísta & $\mathbf{3 5 . 0 7}$ & 7.70 & 28.46 & 11.07 & $6.01 *$ \\
\hline Indisciplinado & 28.09 & 11.09 & 34.89 & 13.09 & $4.65^{\star}$ \\
\hline Sádico & 9.23 & 7.89 & 14.88 & 10.36 & $5.20^{\star}$ \\
\hline Conformista & 47.98 & 9.65 & 36.17 & 12.21 & $9.04^{*}$ \\
\hline Oposicionista & 16.66 & 9.56 & 29.47 & 11.73 & $10.01^{\star}$ \\
\hline Autodestructivo & 14.56 & 10.35 & 32.01 & 12.73 & $12.60^{\star}$ \\
\hline Tendencia límite & 9.54 & 6.07 & 19.32 & 8.14 & $11.63^{*}$ \\
\hline Difusión Identidad & 11.14 & 5.97 & 20.43 & 8.41 & $10.99^{\star}$ \\
\hline Autodevaluación & 15.82 & 10.92 & 31.69 & 13.38 & $10.87^{\star}$ \\
\hline Desaprob. corporal & 7.30 & 7.48 & 13.19 & 7.98 & $6.18^{*}$ \\
\hline Incomodidad sexual & 27.72 & 6.62 & 24.99 & 8.25 & $3.06^{\star}$ \\
\hline Inseguridad pares & 8.34 & 4.88 & 11.31 & 6.41 & $4.43^{\star}$ \\
\hline Insensibilidad social & 27.80 & 8.17 & 26.60 & 10.84 & 1.06 \\
\hline Discordia familiar & 14.15 & 5.74 & 19.61 & 7.61 & $6.89^{*}$ \\
\hline Abuso infantil & 4.84 & 3.41 & 10.40 & 6.33 & $9.99^{*}$ \\
\hline Trastorno alimentario & 8.74 & 8.49 & 16.43 & 8.90 & $7.15^{*}$ \\
\hline Abuso de sustancias & 12.19 & 9.15 & 20.86 & 12.10 & $6.87^{*}$ \\
\hline Predisposición delincuencia & 21.91 & 6.20 & 22.68 & 8.67 & 0.87 \\
\hline Impulsividad & 14.23 & 5.96 & 18.69 & 7.42 & $5.57^{*}$ \\
\hline Ansiedad & 30.49 & 5.97 & 31.29 & 9.68 & 0.88 \\
\hline Depresión & 13.61 & 6.58 & 23.71 & 9.65 & $10.65^{*}$ \\
\hline Tendencia suicida & 5.23 & 5.51 & 14.97 & 8.59 & $11.90^{*}$ \\
\hline
\end{tabular}

${ }^{*} p<0.001$

(introvertido, inhibido y triste), la percepción de sí mismos se caracteriza por la alta devaluación, son propensos a desarrollar trastornos en los comportamientos alimentarios y presentan sintomatología clínica depresiva.

2) Grupo con trastornos internalizantes y alteraciones de la personalidad severas $(n=11)$. Este grupo, al igual que el anterior, presenta puntuaciones elevadas en aquellos patrones de personalidad con características internalizantes y déficits en la polaridad placer. Sin embargo tienen puntuaciones muy elevadas en casi todas las escalas de preocupaciones actuales y en la escala de trastorno borderline de la personalidad. Se trata de un grupo con alteraciones graves de la personalidad; registran puntuaciones elevadas en la escala abuso infantil y en los síndromes clínicos que connotan trastornos depresivos y tendencia suicida. Es el grupo en más alto riesgo psicológico.

3) Grupo Impulsivo $(n=15)$. Estos adolescentes registran conductas antisociales y narcisistas; obtienen la fuente de refuerzo principalmente en sí mismos. Revelan también características de 
personalidad borderline así como una propensión al desarrollo de conductas impulsivas y abuso de sustancias psicoactivas.

4) Grupo sin síndromes clínicos $(n=30)$. Este grupo registra puntuaciones bajas en casi todas las escalas del MACI. Presenta un patrón de personalidad similar a quienes no se encontraban en RA por lo que se lo caracteriza como el de menor riesgo. Se considera que los síntomas autoinformados son transitorios, producto de algún evento vital estresante momentáneo, no hay riesgo de desarrollo de trastornos de personalidad $u$ otro síndrome clínico de importancia.

El análisis de los datos obtenidos indica que no todos los adolescentes considerados en situación de riesgo psicológico presentan los mismos patrones de personalidad, existen importantes diferencias individuales intragrupales. Como paso siguiente se relacionaron estos cuatro perfiles clínicos con las variables edad y sexo. Previamente se recodificó la variable edad en dos categorías (entre 13 y 15 años y mayores de 15 años). Se verificó que estos perfiles eran independientes de las edades de los jóvenes $\left(\chi^{2}(3)=3.13, n s\right)$, y se pudo constatar asociación con la variable género $\left(\chi^{2}(3)=19.90, p<0.001\right)$ : los varones eran más propensos a presentar trastornos impulsivos y las mujeres trastornos internalizantes leves.
Perfiles de satisfacción de adolescentes

Con la finalidad de verificar si los jóvenes de RA tenían un nivel de bienestar diferente de aquéllos con $B R$, se compararon las puntuaciones en la escala BIEPS de ambos grupos. Se pudo constatar que los considerados RA tienen menor nivel de BP en cada una de las dimensiones de la BIEPS: menor control de las situaciones, menor aceptación de sí mismos, mayor ausencia de proyectos y dificultad para establecer vínculos interpersonales (Tabla 2).

Finalmente se llevó a cabo un análisis discriminante para revisar los aspectos del BP más relacionados con adolescentes categorizados RA y RB. Se obtuvo una función discriminante que explica el $23 \%$ de la variación de las puntuaciones del bienestar entre ambos grupos $(\lambda=$ $\left.0.81, \chi^{2}(2)=53.10, p<0.05\right)$. La dimensión proyectos es la que más discrimina entre ambos grupos. Se realizó un segundo análisis discriminante para determinar la existencia de diferencias individuales en la percepción del BP entre los adolescentes que se encontraban en el grupo RA. Se tomaron como variables dependientes las subescalas de la BIEPS y su puntuación total y como variable independiente la pertenencia a cada subgrupo. Se obtuvieron tres funciones discriminantes. La primera de ellas explica un alto porcentaje de la varianza (autovalor $=0.46$; correlación canónica $=0.56$;

Tabla 2. Perfiles BIEPS de estudiantes con alto y bajo riesgo psicológico

\begin{tabular}{|c|c|c|c|c|c|}
\hline \multirow[b]{2}{*}{ Escalas BIEPS } & \multicolumn{2}{|c|}{$\begin{array}{c}\text { Bajo riesgo } \\
(n=222)\end{array}$} & \multicolumn{2}{|c|}{$\begin{array}{l}\text { Alto Riesgo } \\
(n=90)\end{array}$} & \multirow[b]{2}{*}{$t$} \\
\hline & Media & $\mathrm{DT}$ & Media & $D T$ & \\
\hline Total BIEPS & 34.02 & 4.39 & 29.04 & 5.15 & $6.59^{*}(a)$ \\
\hline Control & 10.70 & 1.59 & 9.16 & 2.09 & $5.74^{*}(\mathrm{a})$ \\
\hline Vínculos & 7.86 & 1.29 & 6.76 & 1.54 & $5.88 *(\mathrm{\theta})$ \\
\hline Proyectos & 7.75 & 1.42 & 7.01 & 1.72 & $3.30 *(\mathrm{~b})$ \\
\hline Aceptación & 7.69 & 1.29 & 6.61 & 1.48 & $5.83^{*}(\mathrm{a})$ \\
\hline
\end{tabular}

Nota: (a) tamaño del efecto alto; (b) tamańo del efecto moderado

${ }^{*} p<0.001$ 
$86 \%$ de la varianza). El análisis discriminante permitió verificar que la sensación de control ( $r$ con la función $=0.82$ ) y el tener proyectos ( $r$ con la función $=0.75$ ) son las dimensiones del BP que más diferencian entre los subgrupos de jóvenes en riesgo. Tanto el tener proyectos de vida como la capacidad para controlar situaciones no sólo discriminan entre adolescentes RB y RA sino también entre los diferentes clusters psicopatológicos analizados.

\section{DISCUSIÓN}

Con relación al objetivo principal de esta investigación se puede concluir que los datos presentados confirman que las instituciones educativas son ámbitos adecuados para el desarrollo de tareas de tipo epidemiológico que posibilitan la detección de adolescentes en riesgo. Se considera que el haber podido ubicar un $28 \%$ de estudiantes con problemas psicológicos de importancia clínica da sustento a lo expresado anteriormente. En el marco de las acciones que se desarrollan hasta el presente en los ámbitos de los sistemas de salud y educación públicas de la ciudad de Buenos Aires puede afirmarse que el espacio educativo está muy desaprovechado por los profesionales que manifiestan estar interesados en la prevención y asistencia clínica y rehabilitación de la salud psíquica de los adolescentes.

Los estudiantes identificados en situaciones de RA presentaban básicamente trastornos de personalidad con características borderline, habiéndose comprobado que no conforman un grupo clínicamente homogéneo sino que existen importantes diferencias interindividuales. Se han encontrado trastornos internalizantes con y sin graves alteraciones de la personalidad así como trastornos relacionados con el control de los impulsos, no asociados con la edad de los adolescentes pero sí con la variable género. Los adolescentes considerados de $\mathrm{RB}$ tienden a dramatizar más sus conflictos, están más centrados en si mismos (alto individualismo), son personas en las que predominan las actitudes conformistas y la insensibilidad social y parecen estar más disconformes con su sexualidad.

Este estudio ha permitido constatar que el BP aparece asociado con situaciones de bajo riesgo. Los perfiles de quienes son considerados en RA muestran trastornos internalizantes graves así como bajo control de los impulsos. En términos generales las dimensiones del BP que diferencian más entre los adolescentes RA de los RB son el control de situaciones y la existencia de proyectos de vida. Los estudiantes $R A$ informan sobre la existencia de dificultades para el manejo de los asuntos de la vida diaria, parecen no darse cuenta de las oportunidades que les presenta el medio y se consideran incapaces de modificar el ambiente.

Se considera importante haber verificado que la dimensión relacionada con la elaboración de un proyecto de vida es una de las que más diferencia entre los grupos en RA y RB. Para quienes trabajan con adolescentes, tanto desde un abordaje clínico como educativo, estos datos plantean la necesidad de tener como meta de las intervenciones profesionales la construcción del proyecto de vida personal, el desarrollo del sentido de autocompetencia y el logro de estrategias de afrontamiento que posibiliten un mejor control del ambiente.

La escala BIEPS, integrada por sólo trece items, es un instrumento válido, fiable, práctico y eficaz para identificar sujetos en riesgo (screening). A partir del estudio realizado puede afirmarse que una baja puntuación total en la BIEPS puede dar cuenta de la existencia proba- 
ble de problemática psicológica cuya especificidad y gravedad deberán ser diagnosticadas. El inventario clínico de Millon para adolescentes (MACI) ha resultado una técnica de diagnóstico válida y su uso en tareas similares a las informadas en el presente trabajo es altamente recomendable.

A partir de los estudios realizados y en curso sobre BP, se considera que es necesario desarrollar investigaciones que tengan en cuenta dicho constructo en el contexto de la diversidad cultural que caracteriza nuestro mundo actual tan globalizado en lo económico. En este sentido compartimos las sugerencias de Triandis (2001) quien sostiene que la cultura es a la sociedad lo que la memoria es a los individuos y, por lo tanto, es imposible dejar de tener en cuenta el estudio de las dimensiones culturales que intervienen en la construcción de la noción de BP. Si el BP ha sido definido en términos de valoraciones que las personas hacen en función de haber o no logrado ciertas metas o expectativas, es indudable que éstas se incorporan a partir de procesos de socialización primaria que se sostienen en valores y creencias planteadas como social y culturalmente válidas. No cabe duda que las diferencias encontradas referidas a mayor presencia de problemática depresiva (internalizante) en mujeres y las relativas al manejo y control de los impulsos(externalizantes) en la muestra masculina suponen la mediación de variables relacionadas con pautas y expectativas culturales.

Los datos analizados en el presente trabajo, en términos generales, muestran que el BP está asociado con la ausencia de síntomas, síndromes o patrones de personalidad con significación clínica. Sin embargo, es importante tener presente que esos mismos datos están indicando que los adolescentes que no presentan riesgo psicopatológico o tienen puntua- ciones altas en BP son mucho más individualistas, tienden a buscar protección y ayuda mediante el uso de estrategias que suponen manipulación y seducción (dramatizan), se reconocen más conformistas, lo que según el propio Millon supone altos niveles de represión acompañados por sentimientos de ambivalencia, reconocen tener sentimientos confusos respecto a su sexualidad, miedo a dialogar sobre sus identidades sexuales en proceso de construcción y se muestran como personas más indiferentes hacia lo que sientan o hagan los demás (insensibilidad social). ¿Es probable encontrar datos semejantes si replicamos el estudio con estudiantes adolescentes que viven en contextos culturales y sociales diferentes al nuestro? ¿Puede afirmarse que el BP se logra siendo individualistas, insensibles a las problemáticas sociales, manipuladores y seductores para conseguir apoyo emocional y algo confusos en temas referidos a la sexualidad? Es de esperar que estudios futuros puedan dar respuesta a numerosas preguntas que la realización del trabajo que presentamos nos ha planteado y para las cuales aún no tenemos todas las respuestas deseadas.

\section{REFERENCIAS BIBLIOGRÁFICAS}

Aberastury, A., y Knobel, M. (1971). Adolescencia Normal. Buenos Aires: Paidós.

Argyle, M. (1987). The psychology of happiness. London: Methuen.

Beck, U. (1992). Risk society: Towards a new modernity. London: Sage.

Bernstein, D., Cohen, P., Skodol, A., Bezirganinan, S., y Brook, J. (1996). Chilhood antecedents of adolescent personality disorders. American Journal of Psychiatry, 1537, 907-913.

Casullo, M.M. (1999). Escala para la Evaluación del Bienestar Psicologico. Buenos Aires: Departamento de Publicaciones de la Facultad de Psicología (Universidad de Buenos Aires). 
Casullo, M.M. (2001). Psicología salugénica o positiva: Reflexiones sobre un nuevo paradigma. Anuario de Investigaciones (pp. 340-346). Buenos Aires: Facultad de Psicología, Universidad de Buenos Aires.

Casullo, M.M., y Castro Solano, A. (1999). El inventario clínico para adolescentes MACI de T. Millon. En R.F. Verthelyi (Ed.), Nuevos temas de evaluación psicológica (pp. 133-145). Buenos Aires: Lugar Editorial.

Casullo, M.M., y Castro Solano, A. (1999). Síntomas psicopatológicos en estudiantes adolescentes argentinos. Aportaciones del SCL-90-R. Anuario de Investigaciones (Facultad de Psicología, Universidad de Buenos Aires), 7, 147-157.

Casullo, M.M., \& Castro Solano, A. (2000). Evaluación del bienestar psicológico en estudiantes adolescentes argentinos. Revista de Psicología (Perú). 18, 35-68.

Casullo, M.M., Góngora, V., y Castro Solano, A. (1998). Adaptación del inventario MACI (Millon Clinical Adolescent Inventory) en Argentina: Un estudio preliminar con estudiantes argentinos. Revista Investigaciones en Psicología, 3, 73-89.

Cicchetti, D., y Cohen, D.J. (1995). Perspectives on developmental psychopathology. En: D. Cichetti y D. Cohen (Eds), Developmental psychopathology: Theory and methods (Vol.1, pp. 3-20). New York: Wiley.

Derogatis, L. (1994). SCL-90-R. Symptom CheckList. Manual. Minneapolis: National Computer Systems.

Diener, E., y Suh, E. (2001).(Eds). Culture and subjective well being. Cambridge: The M.I.T. Press. •

Elliott, G., y Feldman, S. (2000). Capturing the adolescent experience. En: S. Feldman y G. Elliott (Eds), At the threshold: The developing adolescent (pp. 1-13). Cambridge: Harvard University Press.

Fierro, A. y Cardenal, V (1996). Dimensiones de personalidad y satisfacción personal. Revista de Psicología General y Aplicada, 49, 1, 65-81.

Frost, R. (1992).Cardiovascular risk modifcation in college students: Knowledge, attitudes and behaviors. Journal of General Internal Medicine, 7, 317- 320.

Hill, P.(1993). Recent advances in selected aspects of adolescent development. Jour- nal of Child Psychology and Psychiatry, 34, 69-99.

Ingram, R., y Price, J. (Eds) (2001). Vulnerability to psychopathology. New York: The Guilford Press.

Kobasa, S., Maddi, S. y Kahn, S. (1979). Hardiness and health: A prospective study. Journal of Personality and Social Psychology, 42, 168-177

Lemos, S. Fidalgo, A.M., Calvo, P. y Menéndez, L. (1992). Salud mental de los adolescentes asturianos. Psichotema, 4, 21-48

Mc. Whirter, J., Mc Whirter, B. y Mc Whirter, E. (1993). At Risk Youth: A_Comprehensive Response. California: Brooks/Cole Publishing Co.

McCullough, G., Huebner, S. y Laughlin, J. (2000). Life events, self-concept and adolescents' positive subjective well-being. Psychology in the Schools, 37, 281-290.

Millon, T. (1993). Millon Adolescent Clinical Inventory. Manual. Minneapolis: National Computer System.

Pallas, A., Natriello, G. y McDull, E. (1989). The changing nature of the desadvantaged population: Current dimensions and future trends. Educational Research, 18, 1622.

Petersen, A.C., y Leffert, N. (1995). What is special about adolescence. En M. Rutter (Ed), Psychosocial disturbances in young people: Challenges for prevention (pp. 85106). New York: Cambridge University Press.

Ryff, C. (1989). Happiness is everything, or is it? Exploration on the meaning of psychological well-being. Journal of Personality and Social Psychology, 57, 1069-1081.

Ryff, C., y Keyes, C. (1995). The structure of psychological well-being revisited. Journal of Personality and Social Psychology, 69, 719-727.

Schmutte, P., y Ryff, C. (1997). Personality and well being: Reexamining methods and meanings. Journal of Personality and Social Psychology, 73, 549-559.

Schulenberg, J., Maggs, J. y Hurrelmann, K.(1997). Health risks and developmental transitions during adolescence. New York: Cambridge University Press.

Triandis, H.C. (2001).Cultural syndromes and subjective well being. En: E. Diener y E. Suh 
(Eds). Culture and subjective well being (pp. 13-36). Cambridge: The M.I.T.Press.

Veenhoven, R. (1988). The utility of happiness. Social Indicators Research, 20, 333354.

Veenhoven, R. (1995). Developments in satis- faction research, Social Indicators Research, 37, 1-46

Walker, Z. y Towsend, J. (1998). Promoting adolescent mental health in primary care: A review of the literature.Journal of Adolescence, $21,621-634$. 